\title{
Infinite conjugacy classes in groups acting on trees
}

\author{
Yves de Cornulier
}

\begin{abstract}
We characterize amalgams and HNN extensions with infinite conjugacy classes.
\end{abstract}
Mathematics Subject Classification (2000). Primary 20E08; Secondary 20 E45.

Keywords. Amalgams, HNN-extensions, infinite conjugacy classes, FC-center.

\section{Statement of the results}

Throughout the paper, we call free group a non-abelian free group.

We characterize amalgams and HNN extensions with infinite conjugacy classes, thereby answering a question by P. de la Harpe [Ha], Problems 27 and 28. Some particular cases are treated in [HP], $\S \mathrm{V}$, [St], Théorèmes 0.1 and 2.8, and [NS]. Our work also provides information about their normal subgroups not containing any free subgroup, and in particular amenable normal subgroups. Our proofs are essentially geometric.

We call an amalgam $A *_{H} B$ non-trivial if $A \neq H$ and $B \neq H$, and nondegenerate if moreover $H$ has index at least three in either $A$ or $B$. Similarly, if $K$ is a group, $H$ a subgroup, and $\theta: H \rightarrow K$ an injective morphism, we call the HNN extension $\operatorname{HNN}(K, H, \theta)$ non-degenerate if either $H$ or $\theta(H)$ is a proper subgroup of $K$, non-ascending if both are proper subgroups, and strictly ascending if exactly one of the two is a proper subgroup.

Let $G$ be a group. If $g \in G$, then the conjugacy class of $G$ is finite if and only if the centralizer $C_{G}(g)$ has finite index in $G$. With this in mind, it is easy to show that the union of all finite conjugacy classes is a subgroup $\operatorname{FC}(G)$ of $G$, of course characteristic, in which every finitely generated subgroup has finite index centralizer and in particular has centre of finite index.

A group $G$ is called icc (infinite conjugacy classes) if $\operatorname{FC}(G)=\{1\}$.

If $G$ is a group and $N$ a subgroup of $G$, we say that $N$ is $f$-normalized by $G$ if $N$ is normal in $G$ and the action by conjugation of $G$ on $N$ has no infinite orbit. 
Given an amalgam $A *_{H} B$, define $\mathrm{FC}_{A, B}(H)$ as the largest subgroup $N$ of $H$ which is normalized by both $A$ and $B$, and such that the subgroup of $\operatorname{Aut}(N)$ generated by $A$ and $B$ f-normalizes $N$.

We begin with the non-degenerate cases.

Proposition 1. For a non-degenerate amalgam $\Gamma=A *_{H} B$, we have $\mathrm{FC}(\Gamma)=$ $\mathrm{FC}_{A, B}(H)$.

For instance, set $A=B=\mathbb{Z} / 4 \mathbb{Z} \ltimes_{ \pm} \mathbb{Z} / 3 \mathbb{Z}$ and $H=\mathbb{Z} / 3 \mathbb{Z}$. Then $\mathrm{FC}\left(A *_{H} B\right)=\mathbb{Z} / 3 \mathbb{Z}$. This contradicts [NS], Example 3.4, where it is claimed that in a non-degenerate amalgam the FC-centre should coincide with the centre, while here the centre is trivial.

Corollary 2. A non-degenerate amalgam $A *_{H} B$ is not icc if and only if there exists a nontrivial subgroup $M \subseteq H$, normalized by both $A$ and $B$, such that either $M$ is finite, or $M \simeq \mathbb{Z}^{n}$ and the images of $A$ and $B$ in $\operatorname{Aut}(M) \simeq \mathrm{GL}_{n}(\mathbb{Z})$ generate $a$ finite subgroup.

Given an $\mathrm{HNN}$ extension $\mathrm{HNN}(K, H, \theta)$, define $\mathrm{FC}_{K, \theta}(H)$ as the largest normal subgroup $N$ of $K$ contained in $H$ that is invariant under $\theta$ and such that the subgroup of $\operatorname{Aut}(N)$ generated by $K$ and $\theta$ f-normalizes $N$.

Proposition 3. For a non-degenerate $H N N$ extension $\Gamma=\operatorname{HNN}(K, H, \theta)$, we have $\mathrm{FC}(\Gamma)=\mathrm{FC}_{K, \theta}(H)$.

Corollary 4. A non-degenerate $H N N$ extension $\operatorname{HNN}(K, H, \theta)$ is not icc if and only if there exists a nontrivial subgroup $M \subseteq H$, normal in $K$ and invariant under $\theta$, such that either $M$ is finite, or $M \simeq \mathbb{Z}^{n}$ and the images of $K$ and $\theta$ in $\operatorname{Aut}(M) \simeq \mathrm{GL}_{n}(\mathbb{Z})$ generate a finite subgroup.

In the degenerate cases, the FC-centre does not have such a simple description. However we can characterize when the group is icc.

Degenerate non-trivial amalgams are amalgams $\Gamma=A *_{H} B$, where $H$ has index two (and is thus normal) in both $A$ and $B$. In this case, the quotient of $A *_{H} B$ by $H$ is isomorphic to the free product of two cyclic groups of order two, and is denoted $D_{\infty}$ (infinite dihedral group). We denote by $Z$ its unique cyclic subgroup of index 2 , and we define $\Gamma^{+}$as the preimage of $Z$ by the map $\Gamma \rightarrow D_{\infty}$.

Proposition 5. A non-trivial, degenerate amalgam $A *_{H} B$ is icc if and only if $\mathrm{FC}_{A, B}(H)=\{1\}$ and the natural morphism $D_{\infty} \rightarrow \operatorname{Out}(H)$ is injective.

Degenerate HNN extensions are merely semidirect products $\mathbb{Z} \ltimes_{\theta} K$. The following result is very likely to be known, but having no reference we give the (elementary) proof. 
Proposition 6. A degenerate HNN extension $\mathbb{Z} \ltimes_{\theta} K$ is icc if and only if $\mathrm{FC}_{K, \theta}(K)=\{1\}$ and the natural morphism $\mathbb{Z} \rightarrow \operatorname{Out}(K)$ is injective.

Two other characteristic subgroups come into play: $\operatorname{RM}(G)$, the largest amenable normal subgroup of $G$, and $\operatorname{NF}(G)$, the largest normal subgroup of $G$ not containing any free subgroup. We have the inclusions

$$
\mathrm{FC}(G) \subseteq \mathrm{RM}(G) \subseteq \mathrm{NF}(G) \subseteq G .
$$

To see that $\operatorname{NF}(G)$ is well defined, observe that if $N, N^{\prime}$ are normal subgroups of $G$ without free subgroups, then so is $N N^{\prime}$ (as it lies in an extension with kernel $N$ and quotient $N^{\prime} /\left(N \cap N^{\prime}\right)$ ), and if $\left(N_{i}\right)$ is an increasing net of normal subgroups without free subgroups, then its union does not have any free subgroup either.

The question by P. de la Harpe mentioned above is related to the operator algebras of groups. In this context, it is also natural to focus on the condition that the amenable radical is trivial: indeed, it is known (and easy) to prove that if a group $\Gamma$ satisfies $\operatorname{RM}(\Gamma) \neq\{1\}$, then $\Gamma$ is not $C^{*}$-simple (see $[\mathrm{Ha}]$ ), while the converse is probably false but unknown (Question 4 in [Ha]) and known to be true for a large class of "nice" groups (see the survey in [Ha]).

For an amalgam $A *_{H} B$, define $\mathrm{RM}_{A, B}(H)$ as the largest normal subgroup of $H$ which is amenable and normalized by both $A$ and $B$. Similarly define $\mathrm{NF}_{A, B}(H)$, and, for an $\mathrm{HNN}$ extension $\operatorname{HNN}(K, H, \theta)$, define $\mathrm{RM}_{K, \theta}(H)$ and $\mathrm{NF}_{K, \theta}(H)$.

Proposition 7. For a non-degenerate amalgam $\Gamma=A *_{H} B$, we have $\operatorname{RM}(\Gamma)=$ $\mathrm{RM}_{A, B}(H)$ and $\mathrm{NF}(\Gamma)=\mathrm{NF}_{A, B}(H)$.

Proposition 8. For a non-ascending $H N N$ extension $\Gamma=\operatorname{HNN}(K, H, \theta)$, we have $\mathrm{RM}(\Gamma)=\mathrm{RM}_{K, \theta}(H)$ and $\mathrm{NF}(\Gamma)=\mathrm{NF}_{K, \theta}(H)$.

In particular, in both cases, $\mathrm{NF}(\Gamma)$ is contained in $H$. Of course this need not be true in a degenerate amalgam or in an ascending HNN extension: in these cases, if $H$ has no free subgroup, then neither does $G$.

The following is an immediate consequence of Proposition 8.

Proposition 9. For a degenerate amalgam $\Gamma=A *_{H} B$ with $H$ of index two in $A$ and $B$, we have $\operatorname{RM}(\Gamma)=\{1\}$ (resp. $\operatorname{NF}(\Gamma)=\{1\})$ if and only if $\operatorname{RM}(H)=\{1\}$ (resp. $\operatorname{NF}(H)=\{1\}$ ) and the natural morphism $D_{\infty} \rightarrow \operatorname{Out}(H)$ is injective.

Proposition 10. For a non-degenerate ascending $H N N$ extension $\Gamma=\mathbb{Z} \ltimes \lim _{\theta} K$, we have $\operatorname{RM}(\Gamma)=\{1\}$ if and only if there exists no nontrivial normal amenable subgroup $N$ of $K$ such that $\theta(N) \subseteq N$.

Proposition 11. For a degenerate $H N N$ extension $\Gamma=\mathbb{Z} \ltimes_{\theta} K$, we have $\operatorname{RM}(\Gamma)=$ $\{1\}$ if and only $\operatorname{RM}(K)=\{1\}$ and the natural morphism $\mathbb{Z} \rightarrow \operatorname{Out}(K)$ is injective. 
Acknowledgment. I thank Pierre de la Harpe for encouragement and valuable comments.

\section{Proofs}

Consider an amalgam $\Gamma=A *_{H} B$; let it act on its Bass-Serre tree: this is a tree with two given vertices $\alpha, \beta$ with stabilizers $A$ and $B$ respectively, linked by an (oriented) edge $\varepsilon$ with stabilizer $H$. Also, if $\Gamma=\operatorname{HNN}(K, H, \theta)$, its Bass-Serre tree is a tree with one given vertex $\alpha$ with stabilizer $K$ and one given (oriented) edge $\varepsilon$ from $\alpha$ to $\theta(\alpha)$ with stabilizer $H$.

Lemma 12. Let $A *_{H} B$ be a non-trivial amalgam, or $\operatorname{HNN}(K, H, \theta)$ be a nonascending HNN extension. Let it act on its Bass-Serre tree. Then it does not fix any point at infinity.

Proof. Suppose that the amalgam $\Gamma=A *_{H} B$ fixes a point $\omega$ at infinity. Exchanging the roles of $A$ and $B$ if necessary, we can suppose that there exists a geodesic ray $\alpha_{0}=\alpha, \alpha_{1}=\beta, \alpha_{2}, \ldots$ whose limit is $\omega$. This implies that the stabilizer of $\alpha$ fixes the edge $\varepsilon$, i.e., $A \subseteq H$, contradicting that the amalgam is non-trivial.

The case of non-ascending HNN extensions is similar.

The following lemma is contained in [PV], Propositions 2 and 3.

Lemma 13. Suppose that a group $G$ acts on a tree. Suppose that it contains a hyperbolic element and preserves no axis, nor any point at infinity. Then $G$ contains a free subgroup.

Lemma 14. Suppose that $G=A *_{H} B$ is a non-degenerate amalgam, or $G=$ $\operatorname{HNN}(K, H, \theta)$ is a non-ascending $H N N$ extension. Consider a normal subgroup $N$ of $G$. Suppose that $N$ contains a hyperbolic element (for the action on the Bass-Serre tree). Then $N$ contains a free subgroup.

Proof. By Lemma 12, $G$ fixes no point at infinity. Moreover, by the non-degenerateness assumption, it preserves no axis. Hence we can apply Lemma 13.

The following lemma is equivalent to [PV], Proposition 1.

Lemma 15. Let $G$ act on a tree without global fixed point on the 1-skeleton by elliptic isometries. Then $G$ fixes a unique point at infinity.

Proof of Proposition 7. It is immediate that $\mathrm{NF}(\Gamma) \cap H=\mathrm{NF}_{A, B}(H)$ (and the similar assertion holds for $\operatorname{RM}(\Gamma)$ ). Thus it suffices to prove that $\mathrm{NF}(\Gamma) \subset H$. Consider the action of $\Gamma=A *_{H} B$ on its Bass-Serre tree. By Lemma 2, $\mathrm{NF}(\Gamma)$ contains no 
hyperbolic element. By Lemmas 15 and 12, it fixes a point $p$ (either a vertex or the middle of an edge). Since it is normal, it fixes all the points in the orbit of $p$, hence fixes its convex hull, that is, all of the tree. In particular, it fixes all oriented edges and hence $\mathrm{NF}(\Gamma) \subseteq H$.

The proof of Proposition 8 is similar and left to the reader.

Proof of Propositions 1 and 3. It is immediate that $\mathrm{FC}(\Gamma) \cap H$ is equal to $\mathrm{FC}_{A, B}(H)$ (resp. $\mathrm{FC}_{K, \theta}(H)$ ).

If we exclude the case of non-ascending HNN extensions, by Propositions 7 and 8 , $\mathrm{FC}(\Gamma)$ is contained in $H$, and thus the propositions are proved.

Now consider the case of a strictly ascending HNN extension $\Gamma=\mathbb{Z} \ltimes \lim _{\theta} K$. Note that since the extension is strictly ascending, $\mathrm{FC}(\Gamma) \subseteq \lim _{\theta} K$. Suppose that $x \in \mathrm{FC}(\Gamma)$ and $x \in \theta^{n}(K)-\theta^{n+1}(K)$ for some $n \in \mathbb{Z}$. Since the conjugacy classes are finite, there exists $k<\ell$ such that $\theta^{k}(x)=\theta^{\ell}(x)$. It follows that $x=\theta^{\ell-k}(x)$, contradicting our assumption. Thus, $x \in \bigcap_{n \in \mathbb{N}} \theta^{n}(K)$ and in particular $\mathrm{FC}(\Gamma) \subseteq K$, and therefore $\mathrm{FC}(\Gamma)=\mathrm{FC}_{A, B}(H)$.

Proof of Corollary 2. Denote by $G$ the group $A *_{H} B$. Clearly, a subgroup satisfying the condition of the corollary is contained in the FC-centre.

Conversely, suppose that $\mathrm{FC}(\Gamma) \neq\{1\}$. Let $M_{0}$ be the subgroup of $\mathrm{FC}(\Gamma)$ generated by one conjugacy class. This group has a subgroup of finite index $d$ isomorphic to $\mathbb{Z}^{n}$ for some $n$. If $M_{0}$ is finite, set $M=M_{0}$. Otherwise, consider a characteristic subgroup $M$ of finite index in $M_{0}$ isomorphic to $\mathbb{Z}^{n}$ : for instance, take the intersection of all subgroups of index $d$ in $M_{0}$. Then the image of the natural map $G \rightarrow \mathrm{GL}_{n}(\mathbb{Z})$ is finite. (To see that an infinite subgroup of $\mathrm{GL}_{n}(\mathbb{Z})$ has an infinite orbit on $\mathbb{Z}^{n}$, consider the action on the union of orbits of basis elements.) Finally $M \subseteq H$ by Proposition 1 .

The proof of Corollary 4 from Proposition 3 is similar and left to the reader.

It remains to deal with the degenerate cases.

The following elementary well-known lemma is useful. Let $\{1\} \rightarrow N \rightarrow G \rightarrow$ $Q \rightarrow\{1\}$ be an extension of groups. The action of $G$ on $N$ by conjugation provides a map $G \rightarrow \operatorname{Aut}(N)$, and after composition defines a map $G \rightarrow \operatorname{Out}(N)$. This map is trivial on $N$ and therefore factors through a (unique) map $Q \rightarrow \operatorname{Out}(N)$.

Lemma 16. Suppose that the natural map $Q \rightarrow \operatorname{Out}(N)$ is injective. Then for every nontrivial normal subgroup $M$ of $G$, we have $M \cap N \neq\{1\}$.

Proof. If $M$ is a normal subgroup of $G$ satisfying $M \cap N=\{1\}$, then $[M, N]=\{1\}$ and therefore $M$ is contained in the kernel of the map $G \rightarrow \operatorname{Out}(N)$. Thus $M \subset N$ and so $M=\{1\}$. 
Proof of Proposition 5. Consider an amalgam $\Gamma=A *_{H} B$ with $H$ of index two in both $A$ and $B$. Note that $\mathrm{FC}(\Gamma) \cap H=\mathrm{FC}_{A, B}(H)$.

Suppose that $\mathrm{FC}(\Gamma) \neq\{1\}$. If $\mathrm{FC}(\Gamma) \cap H \neq\{1\}$, then $\mathrm{FC}_{A, B}(H) \neq\{1\}$. Otherwise, $\operatorname{FC}(\Gamma) \cap H=\{1\}$, and in particular $[\operatorname{FC}(\Gamma), H]=\{1\}$ because $H$ is normal in $\Gamma$. So the natural morphism $\Gamma \rightarrow \operatorname{Out}(H)$ is trivial on both $H$ and $\mathrm{FC}(\Gamma)$, so the factored map $D_{\infty} \simeq \Gamma / H \rightarrow \operatorname{Out}(H)$ is not injective.

Conversely, if $\mathrm{FC}_{A, B}(H) \neq\{1\}$, then $\Gamma$ is clearly not icc. It remains to prove that if $\rho: D_{\infty} \rightarrow \operatorname{Out}(H)$ is not injective, then $\Gamma$ is not icc.

Let $W$ be the kernel of $\rho$ and $M$ its preimage in $\Gamma$. As $M$ is not contained in $H, W$ is a nontrivial normal subgroup of $D_{\infty}$ and hence contains a normal infinite cyclic subgroup $\langle y\rangle$. Lift $y$ to an element $y^{\prime}$ of $\Gamma$. Since $y^{\prime}$ acts on $H$ by $H$-inner automorphisms, there exists $h \in H$ such that $z=y h^{-1}$ centralizes $H$.

We distinguish two cases.

- $H$ has nontrivial centre. If $u$ is central element of $H$, then $u$ is centralized by $H$ and $z$, hence by a finite index subgroup of $\Gamma$. Accordingly $Z(H) \subseteq \operatorname{FC}(\Gamma)$, so $\Gamma$ is not icc.

- $H$ has trivial centre. Indeed, let $C$ denote the centralizer of $H$ in $\Gamma$; this is an infinite normal subgroup of $\Gamma$. Then $H \cap C=\{1\}$, so that $C$ is isomorphic to a subgroup of $D_{\infty}$. The action of $\Gamma$ on $C$ by conjugation factors through $D_{\infty}$, so there are non-trivial finite $\Gamma$-conjugacy classes in $C$, so $\Gamma$ is not icc.

Proof of Proposition 6. Consider a degenerate HNN extension $\mathbb{Z} \ltimes_{\theta} H$.

Suppose that $\mathbb{Z} \rightarrow \operatorname{Out}(H)$ is injective. Then by Lemma 16 every nontrivial normal subgroup of $\Gamma$ intersects $H$ non-trivially. In particular, if $\mathrm{FC}_{K, \theta}(K)=$ $\mathrm{FC}(\Gamma) \cap H=\{1\}$, then $\mathrm{FC}(\Gamma)=\{1\}$.

For the converse, of course if $\mathrm{FC}_{K, \theta}(K) \neq\{1\}$, then $\mathrm{FC}(\Gamma)$, which contains it, is non-trivial. Suppose that $\operatorname{FC}(\Gamma) \cap H=\{1\}$ and $\mathbb{Z} \rightarrow \operatorname{Out}(H)$ is not injective. Denote by $W$ its kernel and by $M$ its preimage in $\Gamma$. Then, as in the case of amalgams, $Z(H) \subseteq \mathrm{FC}(\Gamma)$, and hence $Z(H)=\{1\}$. So the centralizer of $H$ is a normal, infinite cyclic subgroup of $\Gamma$ and is contained in $\mathrm{FC}(\Gamma)$.

Proof of Proposition 9. Let us deal with the amenable radical, the other case being similar. Since $H$ is normal in $\Gamma, \operatorname{RM}(H) \subset \operatorname{RM}(\Gamma)$. Therefore if $\operatorname{RM}(H) \neq\{1\}$, then $\operatorname{RM}(\Gamma) \neq\{1\}$. If $\operatorname{RM}(H)=\{1\}$ and the map $D_{\infty}=\Gamma / H \rightarrow \operatorname{Out}(H)$ is not injective, let $N$ denote the kernel of the map $\Gamma \rightarrow \operatorname{Out}(H)$. By assumption it is not reduced to $H$. Since $\operatorname{RM}(H)=\{1\}$, the group $H$ has trivial centre, and therefore the action by conjugation provides a well-defined map $N \rightarrow H$ which is the identity on $H$. The kernel of this map is normal in $\Gamma$ and isomorphic to a subgroup of $D_{\infty}$ and hence is amenable.

Conversely if $\operatorname{RM}(H)=\{1\}$ and the map $D_{\infty} \rightarrow \operatorname{Out}(H)$ is injective, then by Lemma 16 we obtain that $\operatorname{RM}(\Gamma)=\{1\}$. 
Proof of Proposition 10. Suppose that there exists a nontrivial normal amenable subgroup $N$ of $K$ such that $\theta(N) \subseteq N$. Then the sequence $\left(\theta^{-n}(N)\right)$ is non-decreasing, so that its union is amenable and clearly normal in $\Gamma$.

Conversely, suppose that $\operatorname{RM}(\Gamma) \neq\{1\}$. Set $R=\operatorname{RM}(\Gamma) \cap K$. Then, clearly, $R$ is normal in $K$, amenable, and $\theta(R) \subseteq R$. So, if $R \neq\{1\}$ we are done. If $R=\{1\}$, then $\operatorname{RM}(\Gamma) \cap{\underset{\lim }{\theta}}_{\theta} K=\{1\}$, but this case is excluded since the HNN extension is strictly ascending.

Proof of Proposition 11. Suppose that $\operatorname{RM}(\Gamma)=\{1\}$. Since $\operatorname{RM}(K) \subseteq \operatorname{RM}(\Gamma)$, this implies that $\operatorname{RM}(K)=\{1\}$. Moreover, it follows that $\Gamma$ has infinite conjugacy classes so that, by Proposition 6, the natural morphism $\mathbb{Z} \rightarrow \operatorname{Out}(K)$ is injective.

Conversely, suppose that the conditions are satisfied. By Proposition $6, \Gamma$ has infinite conjugacy classes. Moreover, $\operatorname{RM}(\Gamma) \cap K=\{1\}$, hence $\operatorname{RM}(\Gamma)$ is a normal cyclic subgroup, so that $\operatorname{RM}(\Gamma)=\{1\}$ since $\Gamma$ has infinite conjugacy classes.

\section{Generalization}

Since the results are analogous for amalgams and HNN extensions, it is natural to expect unified statements. This is indeed possible, using graphs of groups.

Recall that a graph $Y$ is defined by the following data:

- a set of vertices $V$,

- a set $E$ of (orientation) edges, with two functions: $t: E \rightarrow V$ (target) and an involutive map $E \rightarrow E, e \rightarrow \bar{e}$ without fixed point, thought as reverting the orientation.

A graph of groups $\mathscr{Z}=(G, Y)$ is defined by the following data:

- a connected graph $Y$,

- a group $G_{v}$ for every $v \in V$; a group $G_{e}$ with $G_{e}=G_{\bar{e}}$ for every $e \in E$; an injective morphism $i_{e}: G_{e} \rightarrow G_{t(e)}$ for every $e \in E$.

To every graph of groups $\mathcal{E}$ is associated its fundamental group $\pi_{1}(\mathscr{E})$ (more correctly, $\pi_{1}(\mathcal{E}, T)$ where $T$ is a maximal tree in the graph).

It is constructed as follows. First define $F(G, Y)$. This is the group generated by (the free product of) all $G_{v}(v \in V)$ and all $e(e \in E)$ subject to the relations $\bar{e}=e^{-1}$ and $e i_{e}(y) e^{-1}=i_{\bar{e}}(y)$ for all $e \in E$ and $y \in G_{e}$. If $T$ is a maximal subtree of $Y$, the fundamental group $\pi_{1}(G, Y, T)$ is the quotient of $F(G, Y, T)$ by the edges in $T$. Up to isomorphism, the group obtained does not depend on the choice of $T$ (see [Se], Chap. 1, §5).

To every graph of groups $(G, Y)$ is associated its Bass-Serre tree (or universal covering) $\tilde{X}=\tilde{X}(G, Y, T)$; this a tree on which the fundamental group $\pi_{1}(G, Y, T)$ acts with quotient $(G, Y)$ (in a suitable sense). Further, there are sections $V \rightarrow V(\tilde{X})$ 
and $E \rightarrow E(\tilde{X})$, denoted $v \mapsto \tilde{v}$ and $e \mapsto \tilde{e}$, such that the stabilizer of $\tilde{v}$ is $G_{v}$ (which is naturally embedded in $\pi_{1}(G, Y, T)$ and the stabilizer of $\tilde{e}$ is $G_{e}$ (more correctly, the subgroup $i_{e}\left(G_{e}\right)$ of $\left.G_{t(e)}\right)$.

We call a graph of groups reduced if there is no edge $e \in E$ satisfying simultaneously the following conditions:

- $t(e) \neq t(\bar{e})$

- $i_{e}$ is an isomorphism.

If we have a non-reduced graph of groups and $e$ is an edge satisfying the conditions above, there is a new graph obtained by removing $t(e)$ (intuitively, we identify $t(e)$ and $t(\bar{e}))$, and, for every edge $e^{\prime}$ such that $t(e)=t\left(e^{\prime}\right)$, define the new target of $e^{\prime}$ as $t(e)$ and replace $i_{e^{\prime}}$ with $i_{e}{ }^{-1} \circ i_{e^{\prime}}$. The fundamental group of the new graph is isomorphic to the old one. Thus, we can replace every finite graph of groups by a reduced one without altering the fundamental group.

We call a graph of groups a bunch if it has only one vertex.

Lemma 17. 1) Suppose that a reduced graph of groups is not among the following exceptions:

- a bunch such that all $i_{e}$ are isomorphisms,

- a bunch with exactly one edge e (and $\bar{e}$ ), such that $i_{e}$ is an isomorphism (but not necessarily $\left.i_{\bar{e}}\right)$.

Let its fundamental group $G$ act on its Bass-Serre tree (universal covering). Then the stabilizers of vertices have no common fixed point at infinity. In particular, $G$ has no fixed point at infinity.

2) If the graph of groups is a bunch with $n \geq 2$ edges, then $G$ does not fix any point at infinity.

Proof. 1) We denote by ordinary letters the vertices or edges in the graph of groups, and by Greek letters the corresponding vertices or edges in the Bass-Serre tree.

Suppose that $\omega$ is a point at infinity fixed by all vertex stabilizers, and let $v_{1}, \ldots$, $v_{n}, \ldots$ be a geodesic ray with end $\omega$, and denote by $\varepsilon_{i}$ the edge joining $v_{i+1}$ and $v_{i}$.

Since the stabilizer of $v_{1}$ fixes $\omega$, it fixes $\varepsilon_{1}$. This means that $i_{\varepsilon_{1}}$ is an isomorphism. Since the graph is reduced, this implies that $\varepsilon_{1}$ is a loop, i.e., $v_{1}=v_{2}$.

Suppose that $v_{1}$ is connected to no other edge than $\varepsilon_{1}$ and its inverse, i.e., we have a bunch with one (non-oriented) edge. Then, by assumption, $i_{\varepsilon_{1}}$ is not an isomorphism, which is a contradiction.

Hence there exists another edge $\varepsilon_{0}$. Let $v_{0}$ be its target and lift $\varepsilon_{0}$ and $v_{0}$ to points $e_{0}$ and $v_{0}$ in the Bass-Serre tree. Then $v_{0}, v_{1}, \ldots$ is a ray with end $\omega$, and therefore the same argument as before shows that $i_{\varepsilon_{0}}$ is an isomorphism. If this is true for all choices of $\varepsilon_{0}$, this means that the graph is a bunch in which all edge morphisms, 
except maybe $i_{\overline{\varepsilon_{1}}}$, are isomorphisms. But taking the path $\varepsilon_{0}$ and then $\varepsilon_{-1}=\overline{\varepsilon_{1}}$, we obtain that $i_{\overline{\varepsilon_{1}}}$ is also an isomorphism, and this is excluded by the assumptions.

2) It remains to consider the case of a bunch with $n \geq 2$ non-oriented edges, in which all edge maps are isomorphisms. In this case, the Bass-Serre tree can be identified with the Cayley graph of the free group $F_{n}$, and the fundamental group contains the left translations, which do not fix any point at infinity (any two of the free generators have no common fixed point at infinity).

Lemma 18. Consider a reduced graph of groups. The Bass-Serre tree is reduced to a line only in the following cases:

- a bunch with exactly one edge e (and $\bar{e}$ ) such that $i_{e}$ and $i_{\bar{e}}$ are isomorphisms;

- a segment with edge e such that the images of $i_{e}$ and $i_{\bar{e}}$ have images of index two.

(Note that this corresponds to degenerate HNN extensions and amalgams.)

The proof is straightforward and omitted.

Proposition 19. Consider a graph of groups and exclude the cases of ascending $H N N$ extensions and degenerate amalgams. Denote by $G$ the fundamental group. Let $N$ be a normal subgroup of $G$ containing no free subgroup. Then the action of $N$ on the Bass-Serre tree is trivial.

Proof. First note that $G$ has no fixed point at infinity by Lemma 17 and preserves no axis by Lemma 18.

Suppose that $N$ contains a hyperbolic element. If it fixes exactly one point at infinity, then this point is $G$-invariant, which is a contradiction. If it fixes exactly two points at infinity, $G$ preserves the axis joining them, which is impossible. Hence, by Lemma 13, $N$ contains a free subgroup, a contradiction.

Hence $N$ is elliptic. If it has no fixed point, it preserves a unique point at infinity which must be fixed by $G$, again a contradiction.

Therefore $N$ fixes a vertex, hence the convex hull of its orbit, that is, all of the tree.

It remains to describe the kernel of the action of the fundamental group $G$ on the Bass-Serre tree.

In the case of an amalgam $A *_{H} B$, this is the biggest subgroup of $H$ which is normal in both $A$ and $B$. In the case of an $\mathrm{HNN}$ extension $\operatorname{HNN}(K, H, \theta)$, this is the biggest subgroup of $H$ which is normal in $K$ and $\theta$-invariant.

In general, this kernel can be described in terms of the data in a graph of group. We need the following elementary definitions: let $X, Y$ be sets. A partial injection $i: X \rightarrow Y$ is a subset of $X \times Y$ whose projections into $X$ and $Y$ are both injective; 
their images are called $\operatorname{Dom}(i)$ and $\operatorname{Im}(i)$. We can of course view $i$ as an bijection of $\operatorname{Dom}(i)$ onto $\operatorname{Im}(i)$. Every partial injection $X \rightarrow Y$ has an inverse $Y \rightarrow X$, which is a partial injection. We can compose partial injections $X \rightarrow Y \rightarrow Z$, although it can happen that we obtain the empty partial injection. In the following, all the partial injections will be partial homomorphisms of groups (that is, partial injections with domain and image subgroups, and defining an isomorphism between these subgroups).

Now let $(G, Y)$ be a graph of groups. To every edge is associated a partial injection $G_{t(\bar{e})} \rightarrow G_{t(e)}$ given by $j_{e}=i_{e} \circ i_{\bar{e}}^{-1}$, with domain $i_{\bar{e}}\left(G_{e}\right)$ and image $i_{e}\left(G_{e}\right)$. Note that $j_{e}=j_{\bar{e}}^{-1}$. Denote by $\mathcal{W}$ the closure of $\left\{j_{e} \mid e \in E\right\}$ under composition.

There exists a unique maximal family of subgroups $W_{v}(v \in V)$ normal in $G_{v}$ such that, for every $w \in \mathcal{W}$ with $w$ a partial injection $G_{v} \rightarrow G_{v^{\prime}}$, we have $W_{v}$ contained in the domain of $w$.

It is then clear that all $W_{v}$ are isomorphic to a single group $W$ : if $v, v^{\prime} \in V$ by connectedness, there exists $w \in \mathcal{W}$ a partial injection $G_{v} \rightarrow G_{v^{\prime}}$, and by uniqueness we must have $w\left(W_{v}\right)=W_{v^{\prime}}$. Fix a base-vertex $v \in V$. Set $W=W_{v}$ and identify all $W_{v^{\prime}}$ to $W_{v}$ through the tree $T$. It is easy to see that this group $W$ corresponds to a single subgroup of the fundamental group $\pi_{1}(G, Y, T)$, which is the kernel of the action in the Bass-Serre tree.

Consider elements $\gamma_{i}$ generating $\pi_{1}(Y, v)$. Every $\gamma_{i}$ induces an automorphism $\alpha_{i} \in \mathcal{W}$ of $W$. Consider the subgroup $\Lambda$ of $\operatorname{Aut}(W)$ generated by all $\alpha_{i}$ and by the action by conjugation of all $G_{v^{\prime}}$ (through the identification $W_{v^{\prime}}=W$ described above).

Proposition 20. Let $(G, Y)$ be a reduced, non-degenerate finite graph of groups and $\Gamma=\pi_{1}(G, Y, T)$. Conjugacy classes of $\Gamma$ contained in $W$ coincide with the orbits of the action of $\Lambda$ in $W$. In particular, $\Gamma$ is not icc if and only if $W$ has a $\Lambda$-invariant nontrivial subgroup which is either finite, or is isomorphic to $\mathbb{Z}^{n}$ and such that the natural morphism $\Lambda \rightarrow \mathrm{GL}_{n}(\mathbb{Z})$ has finite image.

\section{References}

[Ha] P. de la Harpe, On simplicity of reduced C*-algebras of groups. Bull. Lond. Math. Soc. 39 (2007), 1-26. Zbl 1123.22004 MR 2303514

[HP] P. de la Harpe and J.-P. Préaux, Groupes fondamentaux des variétés de dimension 3 et algèbres d'opérateurs. Ann. Fac. Sci. Toulouse Math. (6) 16(2007), 561-589. MR 2379052

[NS] A. C. Naolekar and P. Sankaran, Bounded automorphisms and quasi-isometries of finitely generated groups. J. Group Theory 8 (2005), 515-522. Zbl 1119.20038 MR 2152695

[PV] I. Pays and A. Valette, Sous-groupes libres dans les groupes d'automorphismes d'arbres. Enseign. Math. (2) 37 (1991), 151-174. Zbl 0744.20024 MR 1115748 
[Se] J.-P. Serre, Arbres, amalgames, SL2. Astérisque 46 (1977). Zbl 0369.20013 MR 0476875

[St] Y. Stalder, Moyennabilité intérieure et extensions HNN. Ann. Inst. Fourier (Grenoble) 56 (2006), 309-323. Zbl 1143.20013 MR 2226017

Received October 31, 2007; revised May 8, 2008

Y. de Cornulier, IRMAR, Campus de Beaulieu, 35042 Rennes Cedex, France

E-mail: yves.decornulier@univ-rennes1.fr 